\title{
A Case of Tuberculous Granulomatous Panniculitis without Vasculitis
}

\author{
Yassaman Alipour Tehrany ${ }^{a} \quad$ Laurence Toutous-Trellu $^{b}$ \\ Véronique Trombert $^{\mathrm{a}}$ Jean-Luc Reny ${ }^{\mathrm{a}}$ Gürkan Kaya ${ }^{\mathrm{b}}$ Virginie Prendki ${ }^{\mathrm{a}}$ \\ Departments of ${ }^{\mathrm{a}}$ Internal Medicine, Rehabilitation and Geriatrics and ${ }^{\mathrm{b}}$ Dermatology, \\ University Hospital of Geneva, Geneva, Switzerland
}

\section{Key Words}

Cutaneous tuberculosis · Granulomatous panniculitis · Immunosuppression

\begin{abstract}
We report a case of tuberculous granulomatous panniculitis without vasculitis in an 87-yearold female patient with B-cell chronic lymphocytic leukaemia. One month after starting chemotherapy with chlorambucil and prednisone she presented superficial erythematous plaques on the anterior side of the left leg. Three weeks later erythematous painless deep nodules appeared on the left popliteal fossa and on the left thigh. Cutaneous biopsy revealed granulomatous panniculitis without caseation necrosis or vasculitis. Polymerase chain reaction for Mycobacterium tuberculosis revealed positivity in the skin. The final diagnosis was reactivation of latent tuberculosis (TB) induced by deep immunosuppression associated with chemotherapy and haematological disease. Tuberculous granulomatous panniculitis without vasculitis is a rare presentation of cutaneous TB and may be part of the heterogeneous histopathologic spectrum of erythema induratum of Bazin (nodular vasculitis). Our case shows that the diagnosis of cutaneous TB requires the correlation of clinical findings with histopathology and microbiological tests.

(c) 2015 S. Karger AG, Basel
\end{abstract}

\section{Case Report}

An 87-year-old Swiss woman was admitted to hospital because of increasing dyspnoea. She was suffering from dilated cardiomyopathy, asymptomatic and untreated B-cell chronic lymphocytic leukaemia and cognitive disorders.

On admission, she presented clinical signs of heart failure with pleural effusion on her chest X-ray. A thoracic computed tomography scan showed several enlarged lymph nodes

KARGER 125/s $\quad \begin{aligned} & \text { Yassaman Alipour Tehrany } \\ & \text { Hôpitaux Universitaires de Genève } \\ & \text { Rue Gabrielle-Perret-Gentil 4 } \\ & \text { CH-1205 Genève (Switzerland) } \\ & \text { E-Mail Yasaman.AlipourTehrany @ hcuge.ch }\end{aligned}$


Alipour Tehrany et al.: A Case of Tuberculous Granulomatous Panniculitis without Vasculitis

in the mediastinum and signs of congestive heart failure without any parenchymal lesion. Thoracocentesis revealed a transudate with lymphocyte predominance (67\%). Adenosine deaminase was within the normal range, and both culture and polymerase chain reaction (PCR) of pleural fluid were negative for Mycobacterium tuberculosis (MTB). Flow cytometry showed pleural B-cell chronic lymphocytic leukaemia infiltration, leading to the diagnosis of malignant pleural effusion. Chemotherapy with chlorambucil $0.3 \mathrm{mg} / \mathrm{kg} /$ day ( 5 days/ month) and prednisone $(1 \mathrm{mg} / \mathrm{kg} /$ day) was started, leading to stabilization of the pleural effusion.

One month later, the patient presented with superficial erythematous plaques on the anterior side of the left leg (fig. 1) without fever. Stasis dermatitis seemed the most probable diagnosis and chemotherapy was continued. After 3 weeks, erythematous painless deep nodules appeared on the left popliteal fossa and on the left thigh (fig. 2).

Cutaneous biopsy revealed granulomatous panniculitis without caseation necrosis or vasculitis (fig. 3). Ziehl-Neelsen stain was negative for acid-fast bacilli and skin tissue culture negative for MTB. The result of the T-SPOT.TB interferon-gamma release assay (IGRA) was twice indeterminate. Immunophenotyping for chronic lymphocytic leukaemia was negative and fungal infection was excluded. The patient developed heart failure unresponsive to diuretic treatment and rapid general status alteration. Palliative care was initiated, with the family's agreement, and further pulmonary investigations and blood cultures were not performed. The patient died from the heart failure and autopsy was refused by the family. Urinary culture performed a few days before death because of the appearance of isolated leukocyturia showed the presence of MTB. As PCR for MTB was not initially performed on a fresh cutaneous sample in the absence of acid-fast bacilli, it was done on paraffin-embedded skin samples and shown to be positive post mortem. The final diagnosis was reactivation of latent tuberculosis (TB) with cutaneous and genitourinary localisations. Reactivation was induced by deep immunosuppression associated with chemotherapy and haematological disease.

\section{Discussion}

TB remains a major global health problem. As reported by World Health Organization (WHO) in 2012, an estimated 8.6 million people developed TB and 1.3 million died from the disease.

Cutaneous TB constitutes about 1-2\% of extrapulmonary TB cases [1]. Cutaneous TB lesions show a wide spectrum of morphology, which depends on the endemic zone as well as the patient's age and cellular immune status. Cutaneous TB is classified by bacterial load into multibacillary and paucibacillary forms or according to the mechanism of propagation (direct inoculation, contiguous infection and haematogenous dissemination) [1]. Of all the clinical types, scrofuloderma is the most commonly encountered variant worldwide [1].

We retained the diagnosis of tuberculous granulomatous panniculitis without vasculitis. This presentation is rare and may be part of the heterogeneous histopathologic spectrum of erythema induratum of Bazin (nodular vasculitis). Controversy persists in the literature about whether or not the presence of vasculitis is a histopathologic requirement to establish the diagnosis of erythema induratum of Bazin. In some cases with all the stereotypical clinicopathologic features of erythema induratum of Bazin, serial sections of the sample are unable to demonstrate signs of vasculitis. Therefore vasculitis is not considered as a sine qua non criterion for the diagnosis of erythema induratum of Bazin when other characteristic findings are present $[2,3]$. 
Alipour Tehrany et al.: A Case of Tuberculous Granulomatous Panniculitis without Vasculitis

Erythema nodosum is a frequent type of panniculitis [4]. This diagnosis was eliminated in our patient as the nodules were completely painless with atypical localization. Miliary TB was also suspected because of cellular immunosuppression, but skin biopsy and culture of pleural effusion were not consistent with this diagnosis.

The diagnosis of cutaneous TB requires the correlation of clinical findings with histopathology and microbiological tests. Presence of epithelioid granulomas on biopsy samples is not specific for TB and may also be seen in other conditions such as deep fungal infection, leprosy, sarcoidosis or nontuberculous mycobacteria [1].

IGRA should not be used for the diagnosis of TB in elderly patients as the pre-test probability for TB is high and as the prevalence of indeterminate results increases in individuals aged $>75$ years [5]. Furthermore, the sensitivity of this test is reduced in immunocompromised patients [6].

Mycobacterial culture is the gold standard diagnostic tool. However, it takes about 4 weeks, delaying the diagnosis. Failure to cultivate mycobacteria does not exclude the diagnosis of cutaneous TB, especially in paucibacillary forms $[1,7]$.

Nowadays, the molecular biology approach generates new insights to diagnose TB [8]. Xpert MTB/RIF is an automated PCR that can detect both MTB complex and rifampicin resistance within $2 \mathrm{~h}$ after starting the test. It is a diagnostic tool for pulmonary TB recommended by the WHO with an overall pooled sensitivity and specificity of 90.4 and $98.4 \%$, respectively [9]. However, for extrapulmonary TB, its sensitivity varies widely between different studies, depending on the localization of the TB [10]. In one and the same metaanalysis, the sensitivity of this assay for extrapulmonary TB was $80.4 \%$ and its specificity was $86.1 \%$ [9]. For cutaneous TB no study has yet been carried out.

In our case, PCR analysis of the IS6110 fragment of MTB - an insertion element that exists exclusively within the members of the MTB complex $[7,11]$ - finally led to the diagnosis. Different studies highlighted the contribution of PCR for the diagnosis of TB in granulomatous panniculitis $[4,7,12]$. Chen et al. [4] showed that in a Chinese population PCR was positive in 38.9 and $30.4 \%$ of cases of nodular vasculitis and erythema nodosum, respectively, suggesting that these lesions are associated with active TB. Another study in Taiwan demonstrated that PCR was positive in $56.2 \%$ of cutaneous specimens of cases showing granulomatous inflammation without acid-fast bacilli [7]. Obieta et al. [13] showed on Filipino patients that PCR was a suitable technique for the diagnosis of TB in formalin-fixed, paraffinembedded skin tissue specimens: 1 out of 4 specimens of erythema induratum had positive PCR for the 123-bp segment of the IS6110 gene.

The diagnosis of cutaneous TB in immunosuppressed and elderly people is challenging. It should be systematically considered in the presence of granulomatous panniculitis, even in the absence of vasculitis. In such cases, physicians must not hesitate to perform and repeat cutaneous biopsies and use both histopathology and PCR to confirm the diagnosis.

\section{Statement of Ethics}

The patient's family gave their written informed consent for the publication of this case report.

\section{Disclosure Statement}

The authors declare no conflict of interests. 
Alipour Tehrany et al:: A Case of Tuberculous Granulomatous Panniculitis without Vasculitis

\section{References}

1 Bravo FG, Gotuzzo E: Cutaneous tuberculosis. Clin Dermatol 2007;25:173-180.

-2 Segura S, Pujol RM, Trindade F, Requena L: Vasculitis in erythema induratum of Bazin: a histopathologic study of 101 biopsy specimens from 86 patients. J Am Acad Dermatol 2008;59:839-851.

-3 Schneider JW, Jordaan HF: The histopathological spectrum of erythema induratum of Bazin. Am J Dermatopathol 1997;19:323-333.

4 Chen S, Chen J, Chen L, Zhang Q, Luo X, Zhang W: Mycobacterium tuberculosis infection is associated with the development of erythema nodosum and nodular vasculitis. PLoS One 2013;8:e62653.

5 Beffa P, Zellweger A, Janssens JP, Wrighton-Smith P, Zellweger JP: Indeterminate test results of T-SPOT.TB performed under routine field conditions. Eur Respir J 2008;31:842-846.

-6 Pai M, Denkinger CM, Kik SV, Rangaka MX, Zwerling A, Oxlade O, Metcalfe JZ, Cattamanchi A, Dowdy DW, Dheda K, Banaei N: Gamma interferon release assays for detection of Mycobacterium tuberculosis infection. Clin Microbiol Rev 2014;27:3-20.

7 Hsiao PF, Tzen CY, Chen HC, Su HY: Polymerase chain reaction based detection of Mycobacterium tuberculosis in tissues showing granulomatous inflammation without demonstrable acid-fast bacilli. Int J Dermatol 2003;42:281-286.

8 Negi SS, Basir SF, Gupta S, Pasha ST, Khare S, Lal S: Comparative study of PCR, smear examination and culture for diagnosis of cutaneous tuberculosis. J Commun Dis 2005;37:83-92.

$\checkmark 9$ Chang K, Lu W, Wang J, Zhang K, Jia S, Li F, Deng S, Chen M: Rapid and effective diagnosis of tuberculosis and rifampicin resistance with Xpert MTB/RIF assay: a meta-analysis. J Infect 2012;64:580-588.

10 Lawn SD, Mwaba P, Bates M, Piatek A, Alexander H, Marais BJ, Cuevas LE, McHugh TD, Zijenah L, Kapata N, Abubakar I, McNerney R, Hoelscher M, Memish ZA, Migliori GB, Kim P, Maeurer M, Schito M, Zumla A: Advances in tuberculosis diagnostics: the Xpert MTB/RIF assay and future prospects for a point-of-care test. Lancet Infect Dis 2013;13:349-361.

-11 Coros A, De Conno E, Derbyshire KM: IS6110, a Mycobacterium tuberculosis complex-specific insertion sequence, is also present in the genome of Mycobacterium smegmatis, suggestive of lateral gene transfer among mycobacterial species. J Bacteriol 2008;190:3408-3410.

12 Baselga E, Margall N, Barnadas MA, Coll P, de Moragas JM: Detection of Mycobacterium tuberculosis DNA in lobular granulomatous panniculitis (erythema induratum-nodular vasculitis). Arch Dermatol 1997;133: 457-462.

13 Obieta MP, Obieta MY, Monje VD: Polymerase chain reaction-based detection of Mycobacterium tuberculosis DNA in formalin-fixed, paraffin-embedded skin tissue specimens from Filipino patients. Int J Dermatol 2010;49:470-472.

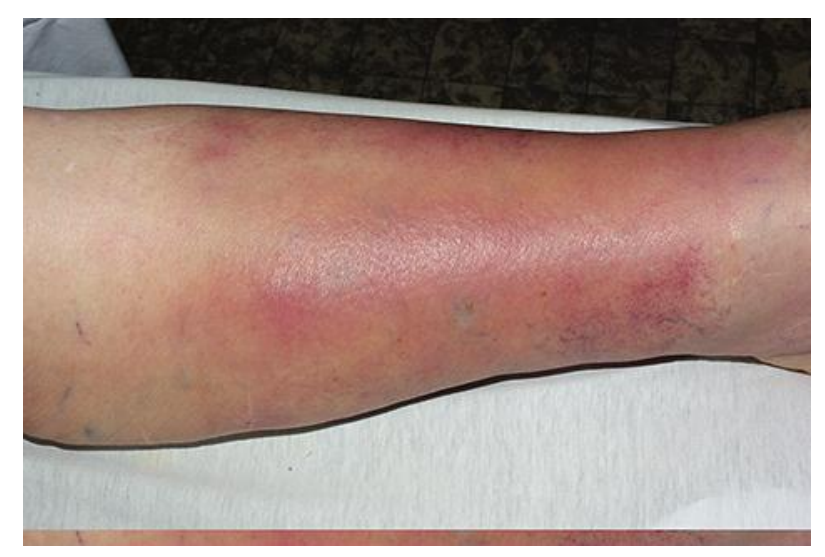

Fig. 1. Superficial erythematous plaques on the anterior side of the left leg. 
Case Reports in
Dermatology

Case Rep Dermatol 2015;7:141-145

DOI: $10.1159 / 000435831$

C 2015 S. Karger AG, Basel

www.karger.com/cde

Alipour Tehrany et al.: A Case of Tuberculous Granulomatous Panniculitis without Vasculitis

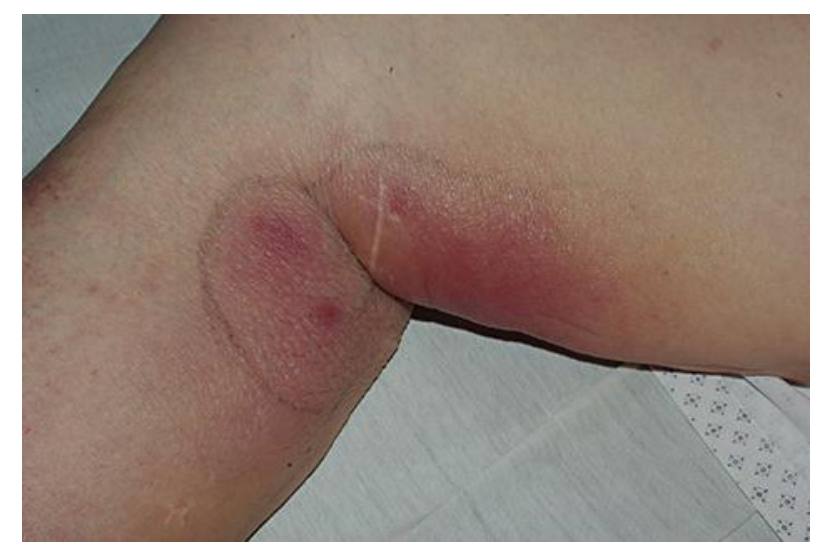

Fig. 2. Erythematous deep nodules on the left popliteal fossa.

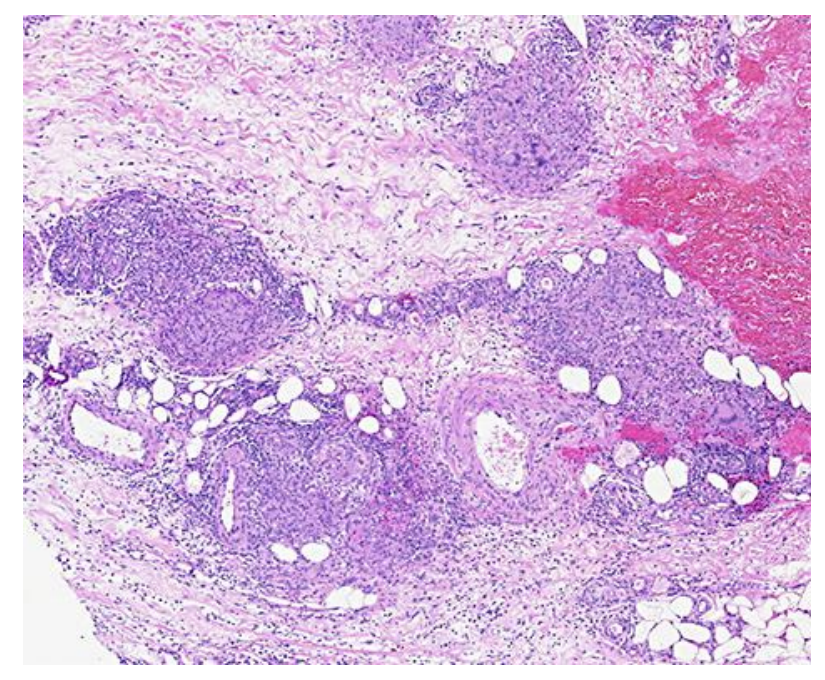

Fig. 3. Septal and lobular granulomatous panniculitis composed of non-caseating granulomatous zones with epithelioid histiocytes and multinucleated giant cells surrounded and infiltrated by lymphocytes. 